\title{
Hiding in Text using Information Integrity Service
}

\author{
Dujan B. Taha \\ dujan_taha \\ @uomosul.edu.iq
}

\author{
Ahmed S. Nori \\ ahmed.s.nori \\ @uomosul.edu.iq
}

Yaseen H. Ismaiel
yaseen-hikmat
@uomosul.edu.iq

Received on:24/11/2008

College of Computer Science and Mathematics

University of Mosul, Iraq

\begin{abstract}
Modern computer networks make it possible to distribute documents quickly and economically. This is because of the decreasing cost of the equipment needed to copy, print, process the information. The widespread adoption of electronic distribution of copyrighted material is accompanied by illicit copying and illicit distribution. This is why people used steganography. Steganography is the art of hiding transmitting data through apparently innocuous carriers in an effort to conceal the existence of the data.

The carrier can be anything used to transfer information, including for example: wood or state tablets, hollow heels, images under stamps, tiny photographs, or word arrangements. Digital carriers include: disk space, disk partitions, text, e-mail, audio, images, and video.

In this paper, we propose a new conceptual framework for text file steganography by using integrity tool. Hash function was used to produce a checksum.

Keywords: Steganography, Hash function.

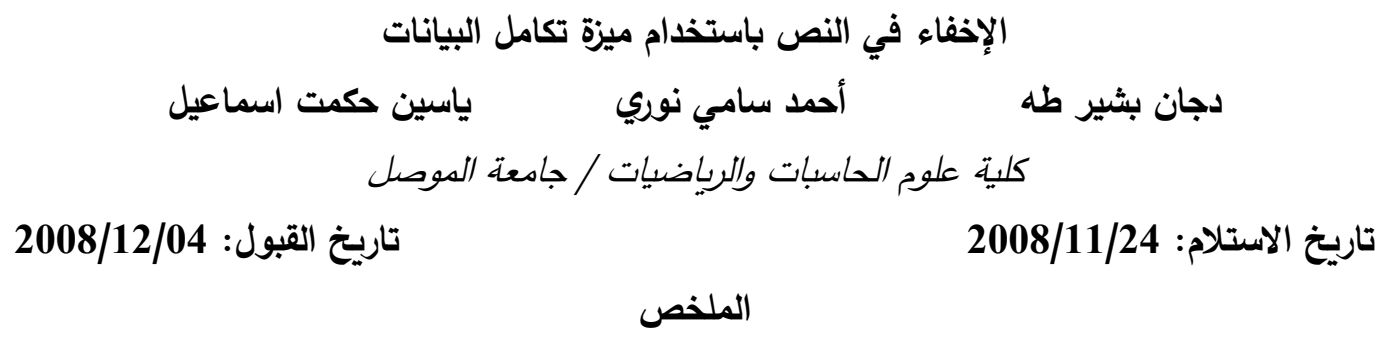

لقد مكنت شبكات الحاسوب الحديثة من توزيع الوثائق بصورة سريعة و اقتصادية ـ يعزى هذا لقلة كلفة

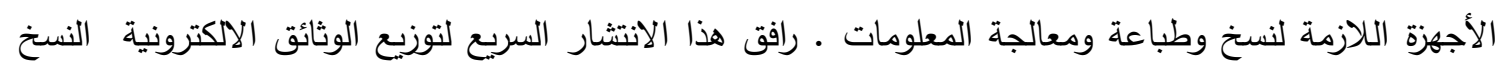

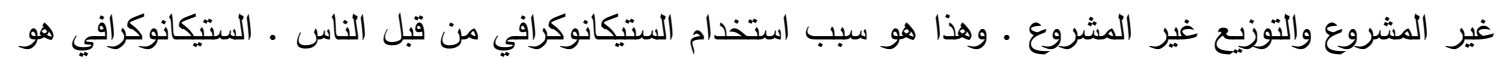

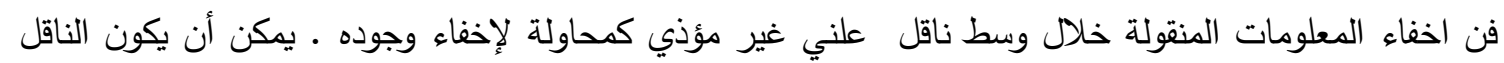

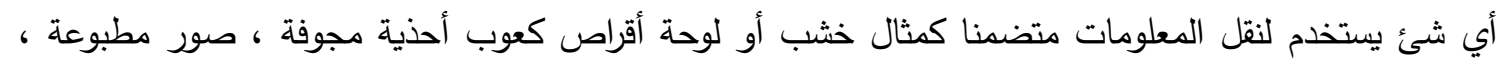

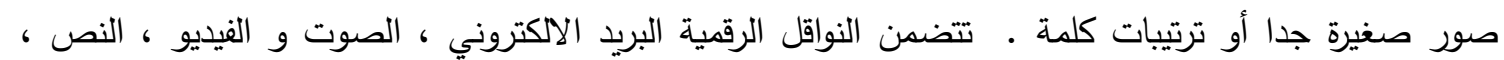

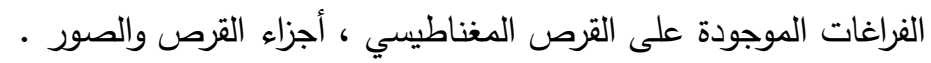
في هذا البحث استخدمت هيكلية جديدة لإخفاء ملف نصي باستخدام ميزة سلامة البيانات (integrity) . تم استخدام الدالة الهاشية (hash function) للحصول على قيمة فحص المجموع. الكلمات المفتاحية: الاخفاء، الدالة الهاشية.
\end{abstract}


: Introduction المقدمة

ستيكانوكرافي (حرفيا الكتابة المغطاة) هي إخفاء الرسائل السرية ضمن رسالة أخرى تبدو غير مؤذية أو

ناقل (Carrier) يمكن أن يكون الناقل أي شيء يستخدم لنقل المعلومات ، متضمنا مثلا خشب أو لوحة أقراص ، كعوب أحذية مجوفة ، صور مطبوعة ، صور صغيرة جدا أو ترتيبات كلمة .تتضمن النواقل الرقمية البريد

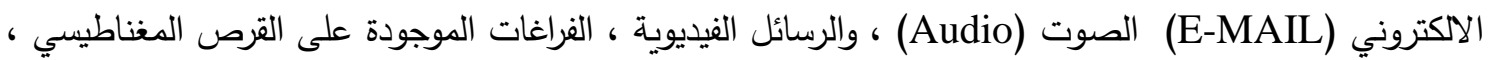
أجزاء القرص الدغناطيسي والصور • الستيكانوكرافي تثبه التثفير لأنها وسائل تؤمن السرية ـ تؤمن الستيكانوكرافي ذلك من خلال إخفاء وجود الاتصال ، بينما يفعل التثفير ذلك من خلال ترميز الرسالة حتى لا يمكن فهمها ـ يمكن مقاطعة الرسالة المشفرة

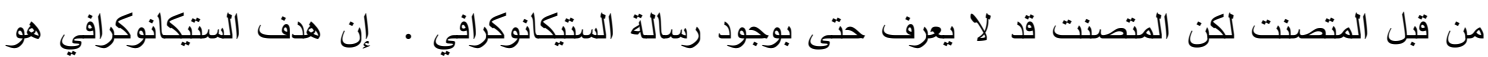

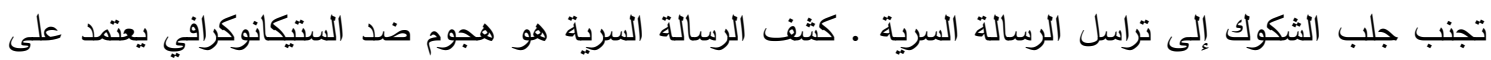
حقيقة أن إخفاء معلومات في وسط رقمي يحور الناقل ويقدم خصائص غير اعتيادية من خلال البيانات المتضمنة

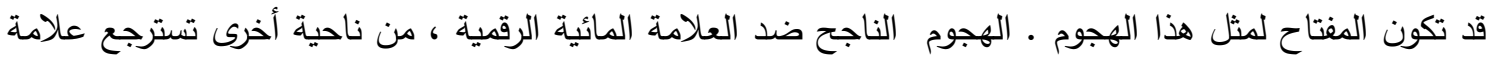
مائية غير مفيدة أو غير مقرؤة [1] • واحدة من أوائل المستتدات التي توصف الستيكانوكرافي هي من إلياذة هيرودتس • في اليونان قديما يكتب

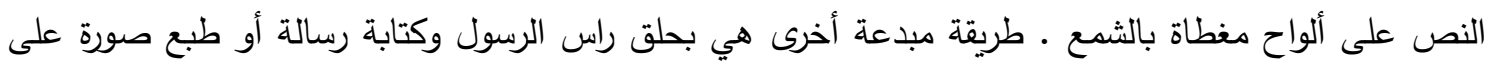

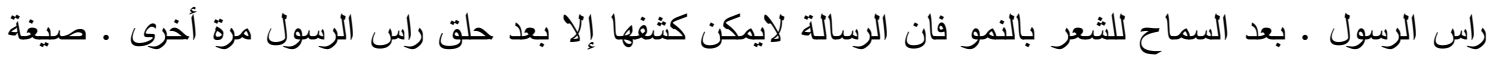

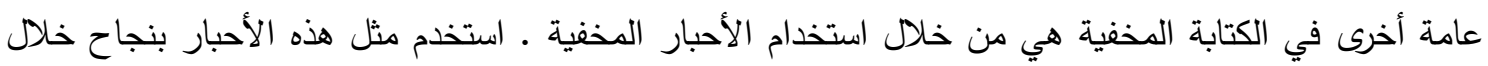

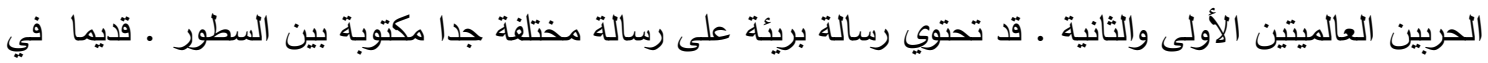
تقنية السيتكانوكرافي في الحرب العالمية الثانية كانت تتألف حصرا من الأحبار المخفية ـ المصادر العامة للأحبار المخفية هي الحليب ، الخل ، عصير الفواكه ، والإدرار (Urine) ، جميعها يغمق لونها عندما تسخن ـ ـ مع تطور

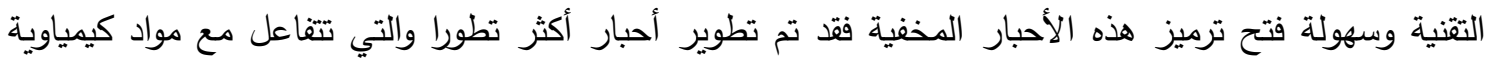

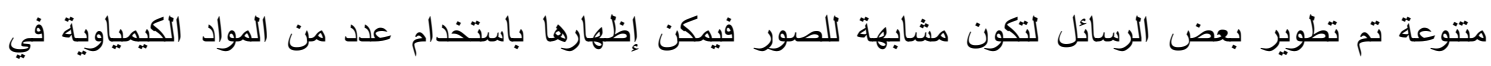

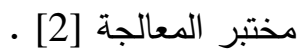

شفرات القيم اللاغية Null Ciphers (رسائل غير مشفرة وتعرف أيضا بالرموز المفتوحة) قد تم استخدامها أيضا حيث تخفى الرسالة الحقيقية في رسالة تظهر أنها بريئة ـ بسبب ظهور العديد من رسائل الرموز المفتوحة ، فان منقيات البريد اكتثفت الاتصالات المشكوك بها ، على كل حال الرسائل التي تظهر أنها بريئة

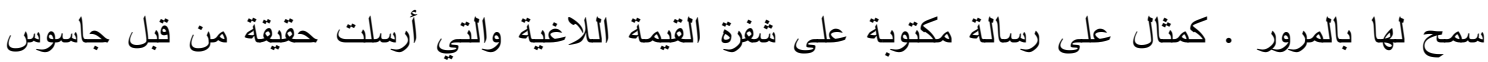

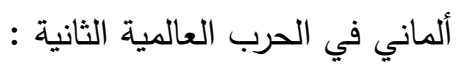

Apparently neutralu $\mathrm{s}$ protest is thoroughly discounted and ignored Isman hard hit.Blockade issue affects pretext for embargo on Byproducts,ejecting suets and vegetable oils.

$$
\text { بأخذ الحرف الثاني من كل كلمة فتظهر الرسالة المخفية التالية : }
$$

Pershhng sails from NYjune 1. 
مثلما تطور كثف الرسائل فقد تم تطوير تقنيات جديدة تستطيع امرار معلومات أكثر وتكون حتى اقل ظهورا. مع طرق عديدة تم اكتثافها ومقاطعتها فقد اتخذ مكتب الرقابة الأمريكي إجراءات نهائية مثل منع تسليم الورد ، الذي يحتوي تواريخ تسليم أحجية الكلمات المتقاطعة وحتى بطاقات التقرير لأنها يمكن أن تحتوي على إهى

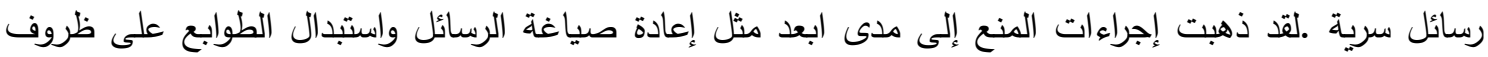

تم في هذا البحث استخدام تقنية أخفاء نص في نص أخر باستخدام طريقة مقترحة تعتمد أسلوب أظهار الرسائل المتضمنة و الوسط الناقل (carrier) سوية ـ تختلف هذه الطريقة عن طرق أخفاء النص السابقة وطريقة

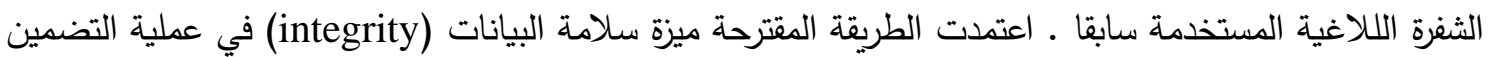
والاسترجاع • استخدمت الدالة الهاشية (function hash) لتطبيق هذه الميزة . 2. 2 تقنيات الإخفاء في النص : يكون عادة من السهل جدا معرفة النسخ الأصلية للكتب من الكتب المستتسخة لان النوعية تكون مختلفة

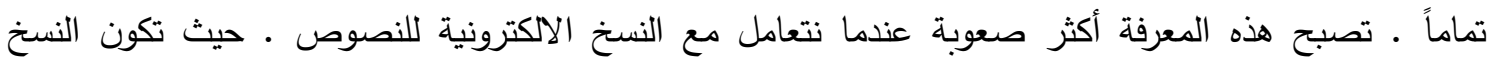
متطابقة ومن المستحيل معرفة ما إذا كانت النسخ أصلية أو مستنسخة ـ فلتضمين معلومات ، مثل العلامة المائية، داخل مستتد تستطيع بكل سهولة تحوير بعض خصائص المستند. يمكن أن تكون هذه تثكيل النص أو خصائص النص

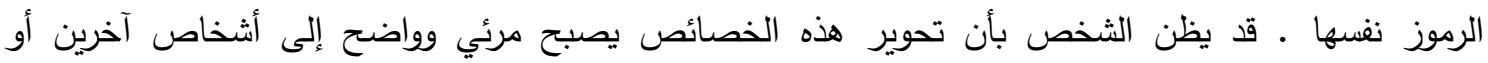

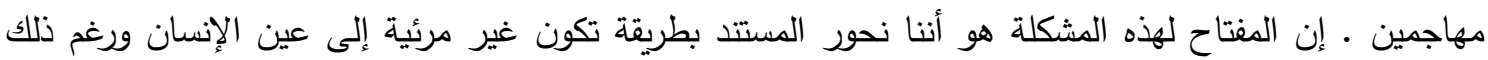

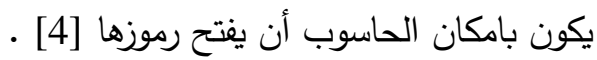

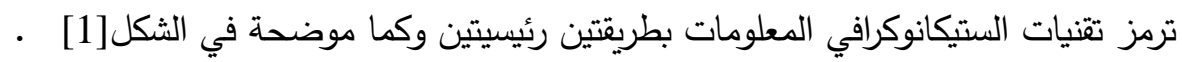

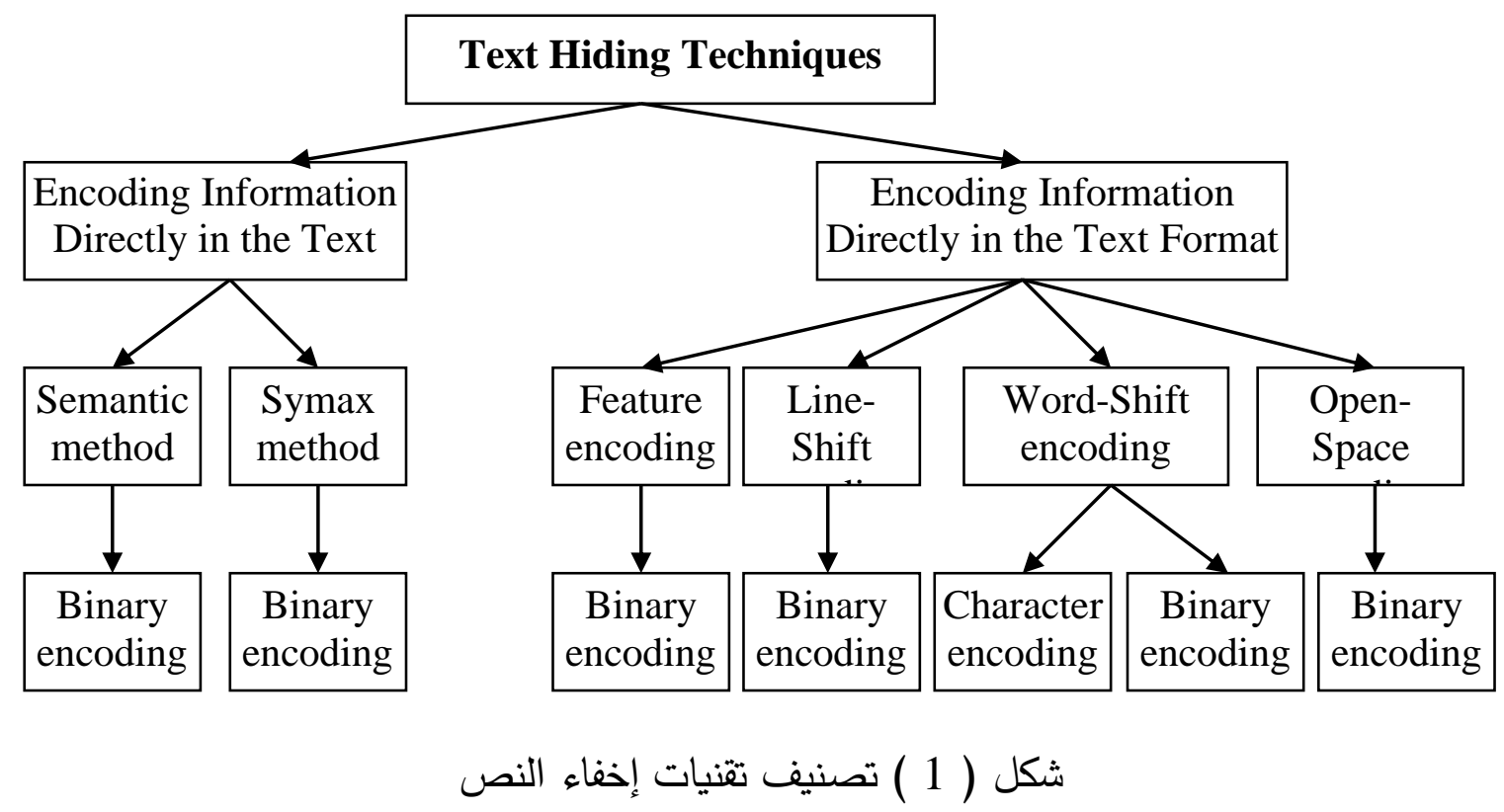


* يمكن توضيح طرق ترميز المعلومات لتحقيق الستيكانوكرافي والموضحة بالثكل [1] بالنقاط التالية

أ. طريقة النحو Syntax Method : تستخدم هذه الطريقة الترقين Punctuation والإلقاء Diction وتركيب النص بدون تغيير المعنى بصورة ملحوظة .

ب. طريقة دراسة معاني الكلمات Semantic Method : تتضمن هذه الطريقة تغيير الكلمات نفسها و انها تخصص قيمتين مترادفة رئيسية او ثانوية . ج. طريقة الفراغ المفتوح Open Space Method : تدل هذه الطريقة على الترميز خلال معالجة الفراغ الأبيض

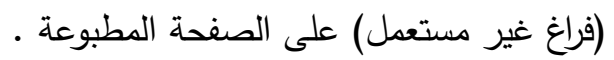

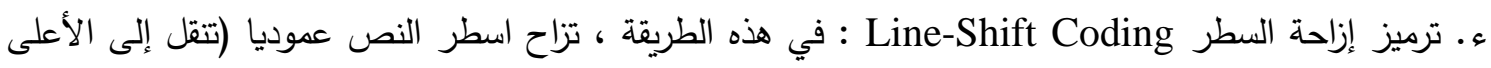

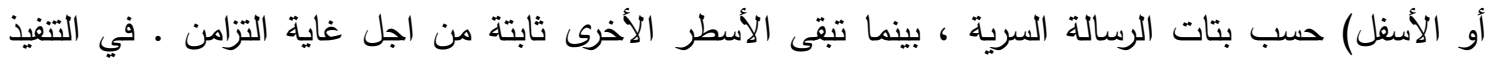

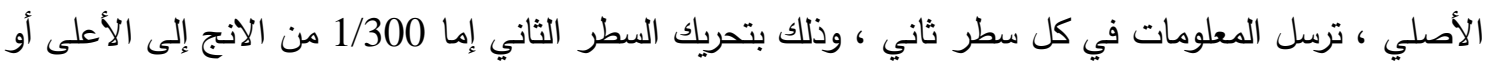

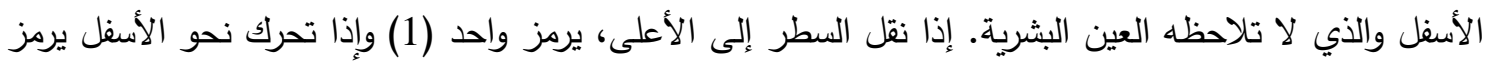

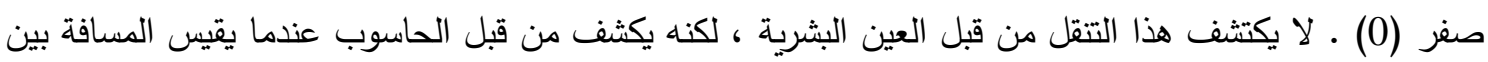

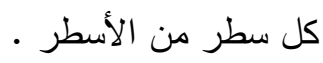

هـ. ترميز إزاحة-الكلمة Word-Shift Coding : في هذه الطريقة ترمز الكلمات الرمزية في المستتد من خلال إزاحة المواقع الأفقية أو العمودية للكلمات ضمن اسطر النص ، بينما يدام ظهور الفراغات الطبيعية ـ تكون هذه

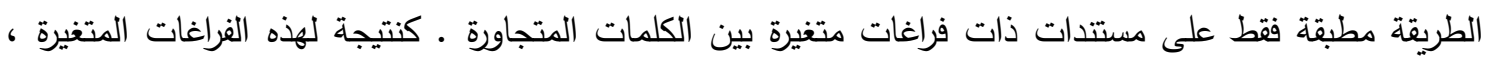

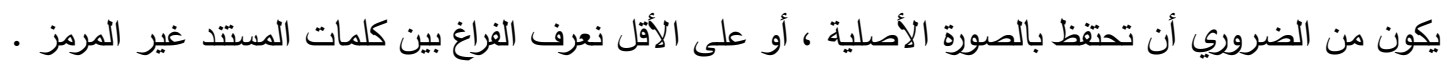

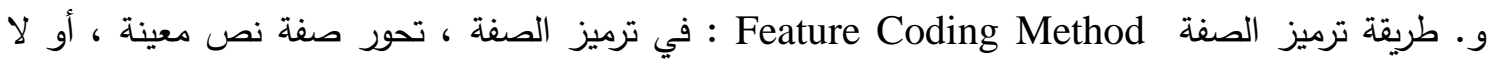

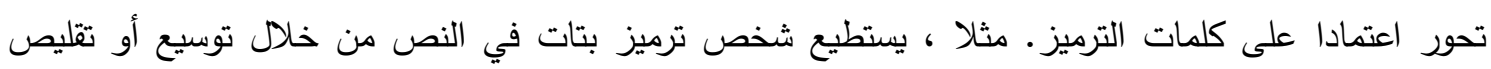

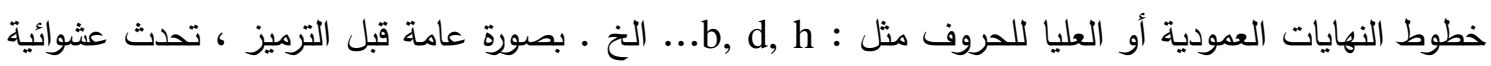
للصفة ـ هكذا ، أطوال نهاية الخط للرمز تطول أو تقصر عشوائيا ، بعد ذلك تحور مرة ثانية لترميز البيانات

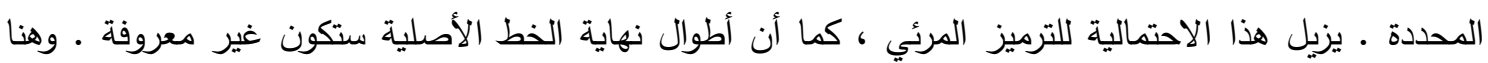

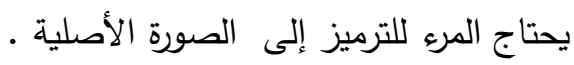

3. MD5 خوارزمية ملخص الرسالة

تم تطوير خوارزمية ملخص الرسالة MD5 من قبل رون رايفس Ron Rivest وهو احد مطوري خوارزمية التثفير غير المتناظر RSA . كانت خوارزمية MD5 هي من أكثر خوارزميات الهاش الأمنية المستخدمة إلى قبل سنين قليلة حيث ظهر الاهتمام بتحليل الثفرة وخاصة هجوم القوة الوحشية (Brute-Force) ـ تأخذ الخوارزمية إخخال الرسالة ذات الطول المختلف وتتتج كإخراج ملخص رسالة ذو طول 128 بت. تتم عملية

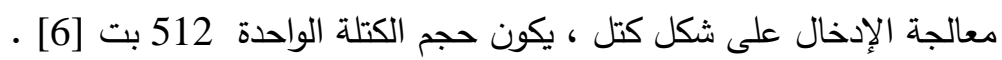
يوضح الثكل (2) المعالجة الكاملة لرسالة لإنتاج الملخص (digest) ـ يتبع هذا الهيكلة العامة . تتألف المعالجة من الخطوات التالية : 
1. إلحاق البتات المبطنة : يتم تبطين الرسالة حتى يكون طولها بالبتات محول إلى (الطول=448 mod 512) هكذا ، يكون طول الرسالة المبطنة هو 64 بت اقل من العدد الذي يكون مكرر إلى 512 بت ـ ويضاف التبطين

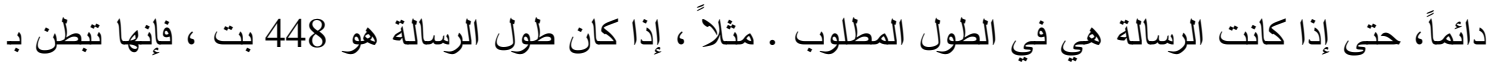

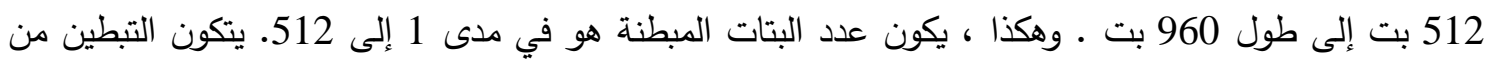

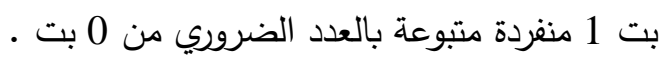

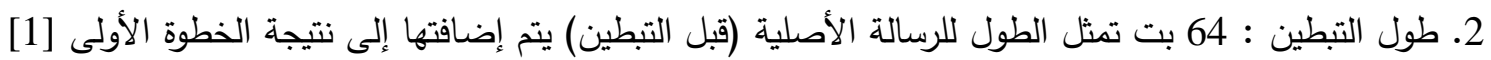
(البايت الأقل أهية أولا). إذا كان الطول الأصلي هو اكبر من 264 ، فيستعمل فقط 64 بت الأقل أهمية من الطول. هكذا، يحتوي الحقل على طول الرسالة الأصلية موديولو 264 .

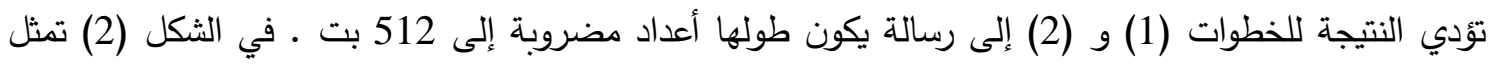
الرسالة الموسعة على شكل سلسلة من كتل 512 بت Y0' Y1' YL-1 حتى يكون الطول الكلي للرسالة
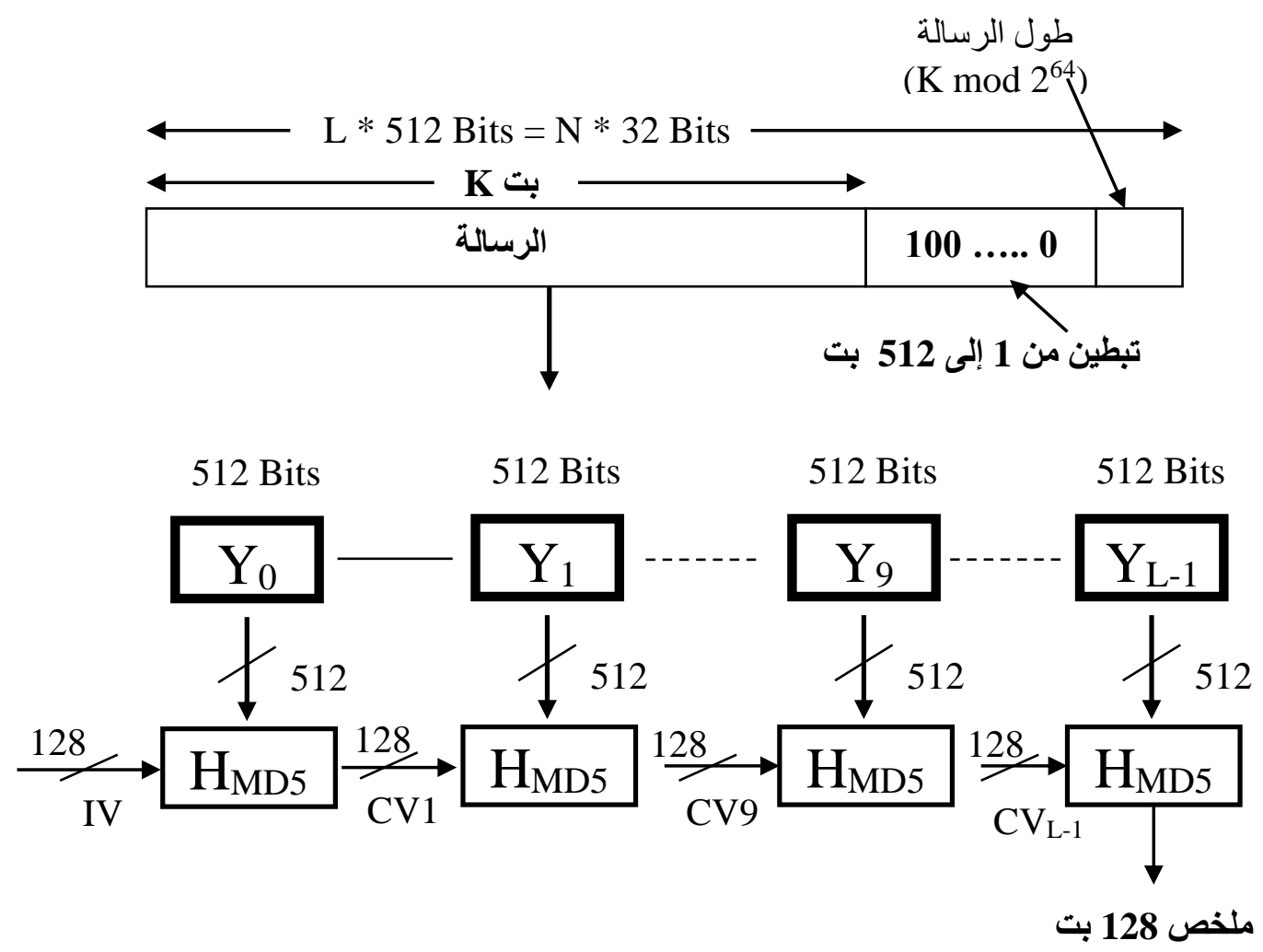

MD5 شكل( 2) توليد ملخص رسالة باستخدام

3. إنشاء المساحة الخزنية MD. مساحة خزنية طولها 128 بت تستعمل لخزن النتائج الوسطية والنهائية لدالة الهاش • يمكن تمثيل هذه المساحة الخزنية كمسجلات ذات طول 32 بت (D،C،B،A).

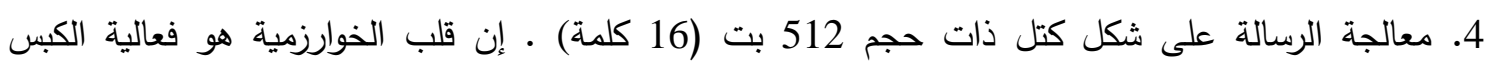
(Compression) 
5. بعد معالجة جميع L ذات 512 بت في الكتلة ، فأن الناتج من مرحلة Lth هي ملخص الرسالة ذو طول 128 بت

تكمن قوة MD5 بأنه يمتلك خاصية أن كل بت في رمز الهاش هي دالة لكل بت في الإدخال. إن الإعادة المعقدة للوظائف الأساسية (I، H، G،F) هي جولات أربعة لها نفس الهيكلة ولكن كل واحدة تستخدم دالة

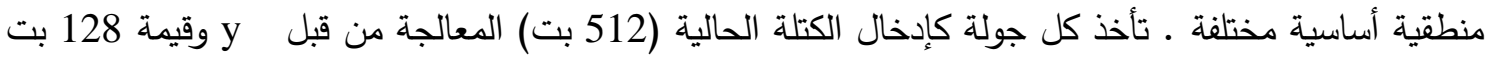
في المساحة البينية وتحديث محتويات المساحة البينية ـ تتتج نتائج ممزوجة بصورة جيدة ، لذلك من غير الممكن

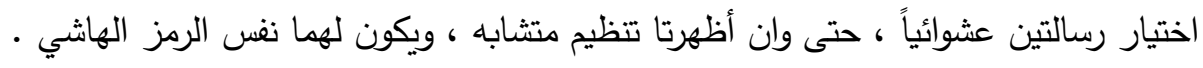

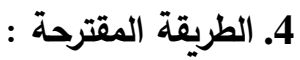
قدمت هذه الطريقة مسار جديد في الستيكانوكرافي • حيث استخدمت النص كناقل لنص آخر سري باستخدام ميزة فحص المجموع (Checksum) • تم إطلاق مصطلح الغربلة (Winnowing) على هذه الطريقة، حيث أنها تغربل النص المتضمن السري عن النص المزيف بالاعتماد على قيمة فحص المجموع الصحيحة .

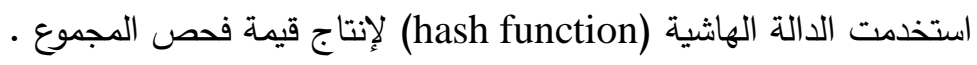
أ. خوارزمية التضمين :

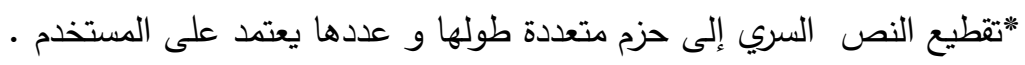

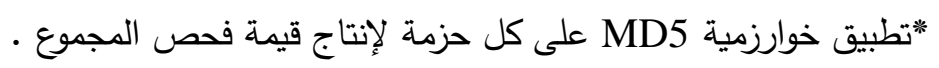

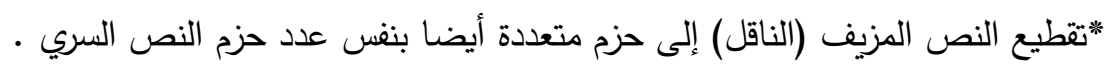
"إعطاء قيم MD5" غير صحيحة لكل حزمة . *دمج النص السري مع النص المزيف وإرسال الرسالة .

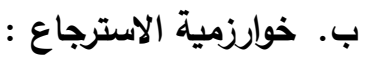

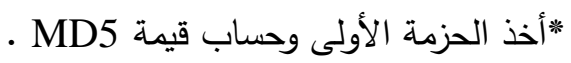

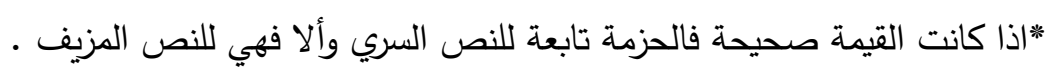
*تكرار الخطوات 1 و 2 لجميع الحزم • مثال تطبيقي : أفترض أن أحمد يريدان يرسل الرسالة النصية التالية الى علي :

"Hi Ali, I'II meet you at 5:00 PM., your friend Ahmed" أولا يجب تقطيع الرسالة إلى حزم وإضافة قيمة فحص المجموع لكل حزمة ـ لو فرضنا بأن الرسالة ستقطع إلى أربع

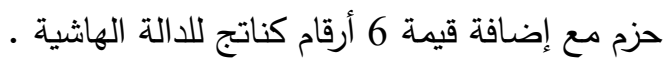
(1, "Hi Ali", 498253 ) (2, "I 'II meet you", 390024 ) (3, "at 5:00 PM" , 759241) (4, "Your friend Ahmed", 258133). في الخطوة الثانية يتم اختيار الوسط الناقل لها وهو الرسالة المزيفة التي تبدو كالتالي : 
تقطع الرسالة الناقلة إلى حزم أيضا وتعطى قيم خاطئة كناتج الدالة الهاشية ـ تضمن الرسالة الأصلية بها حيث تضاف كل حزمة مزيفة قبل أو بعد الحزم الأصلية ـ يظهر الثكل النهائي لها كالتالي :

(1, " Hi Ali", 498253 )

(1, " Hi Suzan", 57801 )

( 2, " I' II call you", 533966 )

(2, " I' II meet you", 390024 )

$(3, "$ at 5:00 PM", 759241 )

(3, " tomorrow", 707224 )

(4, " your brother Ahmed", 539421 )

(4, " your friend Ahmed" 258133 )

5 5

قدمت هذه الطريقة مسار جديد للستيكانوكرافي • تستخدم الطريقة المقترحة وسط نصي لتضمين رسالة

نصية باستخدام الطريقة الدالة الهاشية للحصول على قيمة البصمة أو فحص المجموع و امتازت بما يلي : نسبة التضمين ( data rate) عالية جدا وتعتمد على حجم الغطاء ( الوسط النصي الناقل) فكلما كبر حجم الغطاء

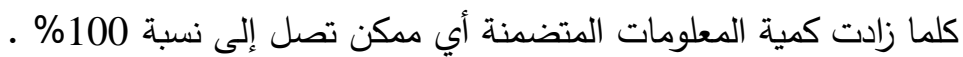
يمكن خزن الرسالة النصية بصيخ مختلفة دون أي تدمير مؤذي لها , لان فيه عملية التضمين لا تعتمد على شكل

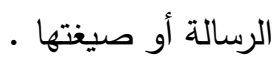
إن هدف الكتابة المخفية هو تجنب جلب الثك إلى تراسل الرسالة المخفية ـ لذلك يبقى غير مكتشف ـ إذا أرتفع الثك يصبح الهدف فاشل ـ الهجمات والتحليل على النص تأخذ المراحل التالية : الكشف Detection وهو عادة الخطوة الأولى وهي فقط معرفة أن احد الأشخاص يستخدم قناة اتصال مخفية ـ في الطريقة المقترحة الكثف سوف يكون صعب جدا لان الناقل هو نص واضح و مفهوم ولا يدعو للثك

الاستخلاص Extraction وهي الخطوة التالية في تحليل النص المخفي والتي تفتح ترميز الملف المكتثف

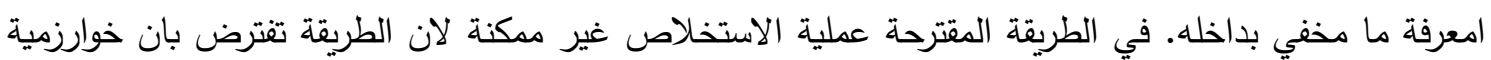
فحص المجموع المستخدمة تبقى سرية بين الباعث والمستلم .

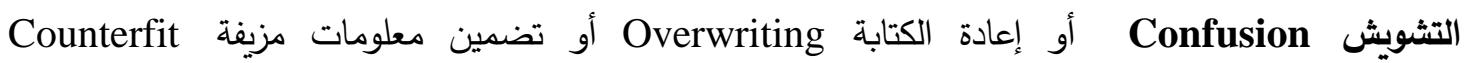
وكية Information فكرة فحص المجموع أو الدالة الهاشية والتي من مميزاتها كثف أي تحوير أو تزييف في الرسالة . التدمير Destruction هي الخطوة الأخيرة وهي الأسهل • إذا لم يستطع المهاجم إثبات نظريته بان هنالك رسالة مخفية في الغطاء فمعنى ذلك أن النظام نظريا يثبت بأنه أمين . كتوصية لتطوير الطريقة ، استخدام الدالة الهاشية ذات المفتاح السري واعتماد سرية المفتاح كوسيلة للتضمين • 


\section{(المصادر}

[1] Al_hamami A.H,information hiding steganography and watermark , university bookshop, alsharaqa,2008 .

[2] doralhy e. denning "information warfare and security", Addison_wesley, 1999.

[3] kazaenbeisser s.,and peticolas f.,"inforation hiding techniques for steganography and digital watermarking ", artech house, 2000.

[4] Neil F. jonson, zoran duric and Sushil Adjoin, "Information Hiding : Steganography and watermarking Attacks and Countermeasures", Kluwer Acadmic publishers, 2001 .

[5] Preneel B. , " the state of cryptographic hash function " , proceeding , eurocryp'ab , 1996 , new york : springer - verlag .

[6] R. , Rivest , 1992 , " the MD5 message Digest Algorithm “ , Internet Engineering task force, RFC 1321 . 